\section{Publisher Correction: Female mice lacking Ftx IncRNA exhibit impaired X-chromosome inactivation and a microphthalmia-like phenotype}

\author{
Yusuke Hosoi ${ }^{1}$, Miki Soma ${ }^{1}$, Hirosuke Shiura ${ }^{1,3,6}$, Takashi Sado (1) ${ }^{4}$, Hidetoshi Hasuwa ${ }^{5,7}$, Kuniya Abe ${ }^{3}$, \\ Takashi Kohda1,6, Fumitoshi Ishino ${ }^{1} \&$ Shin Kobayashi (1) ${ }^{1,2}$
}

Correction to: Nature. Communications.; https://doi.org/10.1038/s41467-018-06327-6, published online 20 Sep 2018.

In the original HTML version of this Article, the affiliation details for Hirosuke Shiura were incorrectly given as: 'Department of Epigenetics, Medical Research Institute, Tokyo Medical and Dental University (TMDU), 1-5-45 Yushima, Bunkyo-ku, Tokyo, 113-8510, Japan; Technology and Development Team for Mammalian Genome Dynamics, RIKEN BioResource Research Center, 3-1-1 Koyadai, Tsukuba, Ibaraki, 305-0074, Japan; Present address: Faculty of Life and Environmental Sciences, University of Yamanashi, 4-4-37 Takeda, Kofu, Yamanashi, 400-8510, Japan'. The correct affiliations are: 'Department of Epigenetics, Medical Research Institute, Tokyo Medical and Dental University (TMDU), 1-5-45 Yushima, Bunkyo-ku, Tokyo 113-8510, Japan; Technology and Development Team for Mammalian Genome Dynamics, RIKEN BioResource Research Center, 3-1-1 Koyadai, Tsukuba, Ibaraki 305-0074, Japan; Faculty of Life and Environmental Sciences, University of Yamanashi, 4-4-37 Takeda, Kofu, Yamanashi 400-8510, Japan'.

Also, in the original HTML version of this Article, the affiliation details for Hidetoshi Hasuwa were incorrectly given as: 'Research Institute for Microbial Diseases, Osaka University, Yamadaoka 3-1, Suita, Osaka, 565-0871, Japan'. The correct affiliations are: 'Research Institute for Microbial Diseases, Osaka University, Yamadaoka 3-1, Suita, Osaka, 565-0871, Japan; Present address: Department of Molecular Biology, Keio University School of Medicine, Tokyo, 160-8582, Japan'.

Additionally, in the original HTML version of this Article, the affiliation details for Takashi Kohda were incorrectly given as: 'Department of Epigenetics, Medical Research Institute, Tokyo Medical and Dental University (TMDU), 1-5-45 Yushima, Bunkyo-ku, Tokyo, 113-8510, Japan; Present address: Department of Molecular Biology, Keio University School of Medicine, Tokyo, 160-8582, Japan'. The correct affiliations are: 'Department of Epigenetics, Medical Research Institute, Tokyo Medical and Dental University (TMDU), 1-5-45 Yushima, Bunkyo-ku, Tokyo, 113-8510, Japan; Faculty of Life and Environmental Sciences, University of Yamanashi, 4-4-37 Takeda, Kofu, Yamanashi 400-8510, Japan'.

These have now been corrected in the HTML version of the Article.

Published online: 31 October 2018

Open Access This article is licensed under a Creative Commons Attribution 4.0 International License, which permits use, sharing, adaptation, distribution and reproduction in any medium or format, as long as you give appropriate credit to the original author(s) and the source, provide a link to the Creative Commons license, and indicate if changes were made. The images or other third party material in this article are included in the article's Creative Commons license, unless indicated otherwise in a credit line to the material. If material is not included in the article's Creative Commons license and your intended use is not permitted by statutory regulation or exceeds the permitted use, you will need to obtain permission directly from the copyright holder. To view a copy of this license, visit http://creativecommons.org/licenses/by/4.0/.

(C) The Author(s) 2018

${ }^{1}$ Department of Epigenetics, Medical Research Institute, Tokyo Medical and Dental University (TMDU), 1-5-45 Yushima, Bunkyo-ku, Tokyo 113-8510, Japan. ${ }^{2}$ Molecular Profiling Research Center for Drug Discovery, National Institute of Advanced Industrial Science and Technology, 2-4-7 Aomi, Koutou-ku, Tokyo 135-0064, Japan. ${ }^{3}$ Technology and Development Team for Mammalian Genome Dynamics, RIKEN BioResource Research Center, 3-1-1 Koyadai, Tsukuba, Ibaraki 305-0074, Japan. ${ }^{4}$ Department of Bioscience, Graduate School of Agriculture, Kindai University, 3327-204, Nakamachi, Nara 631-8505, Japan.

${ }^{5}$ Research Institute for Microbial Diseases, Osaka University, Yamadaoka 3-1, Suita, Osaka 565-0871, Japan. ${ }^{6}$ Faculty of Life and Environmental Sciences, University of Yamanashi, 4-4-37 Takeda, Kofu, Yamanashi 400-8510, Japan. ${ }^{7}$ Present address: Department of Molecular Biology, Keio University School of Medicine, Tokyo 160-8582, Japan. The original article can be found online at https://doi.org/10.1038/s41467-018-06327-6. Correspondence and requests for materials should be addressed to S.K. (email: kobayashi.shin@aist.go.jp) 